\title{
Short Communication: Effect of Phantom Parent Grouping and Properties of Deregression for a Low Heritability Trait
}

\author{
T. Mark, W. F. Fikse, U. Emanuelson, and J. Philipsson \\ Interbull Centre, \\ Department of Animal Breeding and Genetics, \\ Swedish University of Agricultural Sciences \\ 75007 Uppsala, Sweden
}

\begin{abstract}
Properties of deregression for a low heritability trait (clinical mastitis in Sweden with heritability of 0.02) and effects of using different definitions of genetic groups in within-country deregression compared with the across-country analysis were studied. The deregression procedure was satisfactory, and the effects of different phantom parent grouping in within-country deregression compared with across-country analysis were negligible here.
\end{abstract}

(Key words: deregression, genetic group, multipletrait across-country evaluation, low heritability)

Abbreviation key: $\mathbf{C M}=$ clinical mastitis; $\mathbf{M A C E}=$ multiple-trait across-country evaluations.

Deregression of national genetic evaluation results was originally proposed to generate dependent variables to use in international genetic evaluations (Goddard, 1985; Schaeffer, 1985). Today's routine international evaluations of dairy sires are based on multipletrait across-country evaluations (MACE; Schaeffer, 1994) and use deregressed national evaluation results as dependent variables. Deregression and MACE have also been used within country to combine results of longevity analyzed with a survival model and traits analysed with linear statistical models (e.g., Larroque and Ducrocq, 1999). Furthermore, deregression has been used to generate dependent variables for detection of quantitative trait loci (e.g., Thomsen et al., 2001).

Concerns have been raised regarding deregression and effects of phantom grouping in MACE and in estimation of genetic correlations for MACE. Madsen et al. (2001) found that genetic correlations were underestimated for low heritability traits $\left(h^{2}=0.04\right)$, especially when traits were subject to strong selection, but also when there was no selection. On the other hand, they

Received January 16, 2002.

Accepted March 28, 2002.

Corresponding author: T. Mark; e-mail: Thomas.Mark@ hgen.slu.se. found that estimates were unbiased when daughter yield deviations were used instead of deregressed evaluation results, and they hypothesized that bias was introduced in the deregression, since the only pedigree information is through sire and maternal grandsire. Deregression has a larger effect on national evaluations when the heritability is low (Rozzi and Schaeffer, 1996, unpublished), and the importance of pedigree information as well as genetic group effects relative to daughter information increases for low heritability traits. It can therefore be questioned whether deregression and effects of phantom parent grouping disturb the international genetic evaluation results for such traits. The aim of this study was to investigate effects of phantom parent grouping on MACE and properties of deregression for a low heritability trait.

Data used for this study included 1275 Holstein bulls with a Swedish national genetic evaluation result for clinical mastitis (CM). National genetic evaluation results for CM from two other Nordic countries and milk somatic cell results from nine non-Nordic countries were also available and used in across-country analysis. The Swedish CM scale was used in this study, since $\mathrm{CM}$ in Sweden was the available trait with the lowest heritability (0.02). Data used in this study were described in more detail by Mark et al. (2002).

National breeding values were deregressed within country using a single-trait sire model (Jairath et al., 1998). Pedigree was traced back two generations, and genetic groups were formed based on birth year, country of first registration, and selection path. Each group was required to have at least 30 phantom parents. This resulted in the formation of 37 genetic groups in the deregression of Swedish CM evaluation results for 168 missing sires, 189 missing maternal grandsires, and 1705 maternal grandams (all maternal grandams were assumed missing).

Deregressed national breeding values were used as dependent variable in three different genetic evaluation runs denoted $\mathbf{I}_{1}, \mathbf{I}_{2}$, and $\mathbf{I}_{3}$, respectively. In the first run $\left(\mathbf{I}_{1}\right)$, the MACE procedure was applied to only $\mathrm{CM}$ in Sweden, i.e., a single-trait run. The purpose of this run 
Table 1. Comparison of national genetic evaluation results $(\mathbf{X})$ with results from MACE applied to only mastitis in Sweden $\left(\mathbf{I}_{1}\right)$, with MACE results using genetic correlations set to zero $\left(\mathbf{I}_{2}\right)$ and with MACE results using estimated genetic correlations $\left(\mathbf{I}_{3}\right) .1275$ bulls with clinical mastitis results in Sweden were considered.

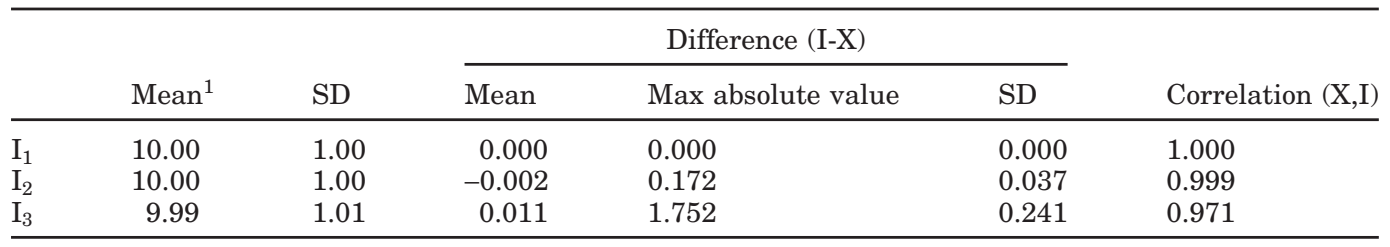

${ }^{1}$ Results were standardized corresponding to mean 10 and standard deviation 1 for $\mathrm{X}$.

was to determine whether the deregression procedure worked according to theory and whether it was unaffected by numerical instabilities, e.g., associated with the low heritability. Genetic groups were identical for $\mathbf{I}_{\mathbf{1}}$ and deregression, i.e., an equivalent set of mixed model equations were considered in both cases. MACE applied to only CM in Sweden was therefore expected to yield exactly the national genetic evaluation results considered as the dependent variable in deregression.

The second $\left(\mathbf{I}_{2}\right)$ and third runs $\left(\mathbf{I}_{3}\right)$ were MACE considering clinical mastitis or milk somatic cell deregressed genetic evaluation results from 12 countries. In $\mathbf{I}_{\mathbf{2}}$, all genetic correlations between countries were set to zero. The purpose of $\mathbf{I}_{2}$ was to study the effect of different phantom parent grouping for foreign bulls in the acrosscountry analysis compared with deregression. The third run $\left(\mathbf{I}_{3}\right)$ was a traditional MACE considering clinical mastitis and milk somatic cell deregressed genetic evaluation results from the 12 countries as different, but correlated, traits $\left(\mathrm{r}_{\mathrm{G}}=0.37\right.$ to 0.97$)$. This run was identical to "Run2" described by Mark et al. (2002). The purpose of this run was to study the impact of correlated information from bulls evaluated in countries other than Sweden on CM in Sweden.

Although definition of genetic groups for $\mathbf{I}_{2}$ and $\mathbf{I}_{\mathbf{3}}$ followed the same principles as in deregression and $\mathbf{I}_{\mathbf{1}}$, the formation of genetic groups for missing ancestors that originated from foreign countries were more elaborate in $\mathbf{I}_{2}$ and $\mathbf{I}_{3}$. In both of the 12-trait MACE runs, 963 missing sires, 1078 missing maternal grandsires, and 51,018 missing maternal grandams were assigned to 198 genetic groups. Definition of genetic groups for missing ancestors of Swedish origin were identical in all three runs, i.e., missing Swedish Holstein sires, maternal grandsires, and maternal grandams were assigned to 1,2 , and 16 unique genetic groups, respectively.

Results from MACE applied on only CM in Sweden $\left(\mathbf{I}_{1}\right)$ showed that deregression was exactly the reverse of MACE (Table 1). This was expected, since this was what the deregression was designed to do, and it confirmed that the deregression worked as it should for CM in Sweden. The low heritability did not cause rounding errors or other instabilities in the deregression that significantly affected the MACE results. The similarity between national evaluation results and $\mathbf{I}_{1}$ was, however, not because deregression did not have an effect. The deregressed national evaluation results deviated substantially from national evaluations (e.g., the standard deviation of deregressed results was 2.2 times higher than for national evaluations, and the correlation between national and deregresed results was 0.90 ).

Some bull evaluations were affected by creating more phantom parent groups for foreign ancestors in MACE than in the deregression, although the average difference was close to zero. The correlation between results from $\mathbf{I}_{1}$ and $\mathbf{I}_{2}$ was 0.999 , and the largest difference was less than 0.2 standard deviation units.

Results from MACE using estimated correlations $\left(\mathbf{I}_{\mathbf{3}}\right)$ were, on average, in good agreement with national evaluation results, although some bulls deviated up to 1.75 standard deviation units due to the extra information considered in traditional MACE. The correlation between results from the two 12-trait MACE runs for the 1275 bulls with a CM evaluation in Sweden was 0.972 . The deviation from unity is explained by correlated information only.

The properties of deregression and phantom parent grouping were hypothesized to be affected by the precision of national evaluation results, but no such effects were found, except that the variation of deregressed national evaluation results decreased as expected with increasing equivalent daughter contributions (results not shown).

The possible bias in estimation of genetic correlations based on deregressed evaluations (Madsen et al., 2001) was not addressed in this study. This study only investigated the properties of the deregressed national evaluation results when used in MACE. In conclusion, the deregression procedure was satisfactory, and effects of less elaborate genetic groups in within-country deregression compared with across-country analysis were negligible for the low heritability trait in question. Further research could investigate effects of phantom grouping and properties of deregression for traits that are subject to strong selection, e.g., production traits. 
The use of deregressed national evaluation results as dependent variable in Interbull evaluations for udder health traits was supported by this study.

\section{REFERENCES}

Goddard, M. E. 1985. A method of comparing sires evaluated in different countries. Livest. Prod. Sci. 13:321-331.

Jairath, L., J. M. C. Dekkers, L. R. Schaeffer, Z. Liu, E. B. Burnside, and B. Kolstad 1998. Genetic evaluation for herd life in Canada. J. Dairy Sci. 81:550-562.

Larroque, H., and V. Ducrocq. 1999. An indirect approach for the estimation of genetic correlations between longevity and other traits. Interbull Bull. 21:128-135.
Madsen, P., M. K. Sørensen, and T. Mark 2001. Validation and comparison of methods to estimate (co)variance components within and across countries from simulated national genetic evaluation results. Interbull Bull. 27:73-79.

Mark, T., W. F. Fikse, U. Emanuelson, and J. Philipsson. 2002. International genetic evaluations of Holstein sires for milk somatic cell and clinical mastitis. J. Dairy Sci. 85:2384-2392.

Schaeffer, L. R. 1985. Model for international evaluation of dairy sires. Livest. Prod. Sci. 12:105-115.

Schaeffer, L. R. 1994. Multiple-country comparisons of dairy sires. J. Dairy Sci. 77:2671-2678.

Thomsen, H., N. Reinsch, N. Xu, C. Looft, S. Grupe, C. Kühn, G. A. Brockmann, M. Schwerin, B. Leyhe-Horn, S. Hiendleder, G. Erhardt, I. Medjugorac, I. Russ, M. Förster, B. Brenig, F. Reinhardt, R. Reents, J. Blmel, G. Averdunk, and E. Kalm 2001. Comparison of estimated breeding values, daughter yield deviations and de-regressed proofs within a whole genome scan for QTL. J. Anim. Breed. Genet. 118:357-370. 